\title{
Identification of the NUP98-PHF23 fusion gene in pediatric cytogenetically normal acute myeloid leukemia by whole-transcriptome sequencing
}

\author{
Marco Togni ${ }^{1}$, Riccardo Masetti ${ }^{*}$, Martina Pigazzi ${ }^{2}$, Annalisa Astolfi ${ }^{3}$, Daniele Zama ${ }^{1}, V^{2}$ lentina Indio ${ }^{3}$, Salvatore Serravalle ${ }^{1}$, \\ Elena Manara ${ }^{2}$, Valeria Bisio ${ }^{2}$, Carmelo Rizzari ${ }^{4}$, Giuseppe Basso ${ }^{2}$, Andrea Pession ${ }^{1}$ and Franco Locatelli ${ }^{5}$
}

\begin{abstract}
The genomic landscape of children with acute myeloid leukemia (AML) who do not carry any cytogenetic abnormality (CN-AML) is particularly heterogeneous and challenging, being characterized by different clinical outcomes. To provide new genetic insights into this AML subset, we analyzed through RNA-seq 13 pediatric CN-AML cases, corroborating our findings in an independent cohort of 168 AML patients enrolled in the AIEOP AML 2002/01 study. We identified a chimeric transcript involving NUP98 and PHF23, resulting from a cryptic $t(11 ; 17)(p 15 ; p 13)$ translocation, demonstrating, for the first time, that NUP98-PHF23 is a novel recurrent (2.6\%) abnormality in pediatric CN-AML.
\end{abstract}

Keywords: NUP98 gene fusions, Pediatric acute myeloid leukemia, PHD domain

\section{Findings}

Childhood acute myeloid leukemia (AML) is a heterogeneous disease with current survival rates of approximately 60-70\%. Cytogenetics, recurrent molecular abnormalities, and early response to treatment are the main factors influencing outcome [1]. However, around $20 \%$ of pediatric AML do not carry any known cytogenetic abnormality (cytogenetically normal-AML or CN-AML). In order to shed light on this subgroup we performed wholetranscriptome sequencing (WTS) in 13 pediatric CNAML cases, corroborating relevant findings in an independent cohort of 168 cases.

Sequencing was performed on a HiScanSQ sequencer (Illumina), and bioinformatic analysis was performed as previously described [2]. In 2 (CN-AML_54, CN-AML_66) out of 13 cases analyzed, we identified a chimeric transcript involving nucleoporin $98 \mathrm{kDa}$ (NUP98) and PHD finger protein 23 (PHF23) genes, resulting from a cryptic translocation $\mathrm{t}(11 ; 17)(\mathrm{p} 15 ; \mathrm{p} 13)$ (Fig. 1a and Table 1). In both cases, we identified an in-frame fusion between NUP98 exon 13 and PHF23 exon 4 (Fig. 1b). To date, the

\footnotetext{
* Correspondence: riccardo.masetti@gmail.com

'Department of Pediatrics, "Lalla Seràgnoli" Hematology-Oncology Unit, University of Bologna, Bologna, Italy

Full list of author information is available at the end of the article
}

cryptic translocation $\mathrm{t}(11 ; 17)(\mathrm{p} 15 ; \mathrm{p} 13)$ has been described only once in an adult AML patient [3], but never in a pediatric AML cohort. Different from what was previously reported by Reader and colleagues [3], in this study the recurrent breakpoint in PHF23 was always identified at the beginning of exon 4 and not within it (Fig. 1a and b).

To assess the incidence of NUP98-PHF23 fusion in pediatric CN-AML, we examined through RT-PCR analysis and Sanger sequencing a validation cohort of 168 AML children enrolled in the AIEOP AML 2002/01 study [4]; one-hundred thirty-nine patients (76 males and 63 females, median age at diagnosis 7.7 years, range 17 days to 17.9 years) were negative for known recurrent genetic abnormalities involving $M L L, C B F B$, and FLT3, while the remaining 29 patients (15 males and 14 females, median age at diagnosis 11.8 years, range 3 to 17.4 years) harbored internal tandem duplication of FLT3 (FLT3-ITD), this latter abnormality being chosen because we previously reported a strong association between NUP98-NSD1 rearrangement and FLT3-ITD [5]. With the exception of FAB M3 (acute promyelocytic leukemia), all the FAB types were represented in the validation cohort. RNA was extracted from fresh bone marrow at diagnosis, and multiplex RT-PCR was used. Sequencing by Sanger method was applied to all cases 


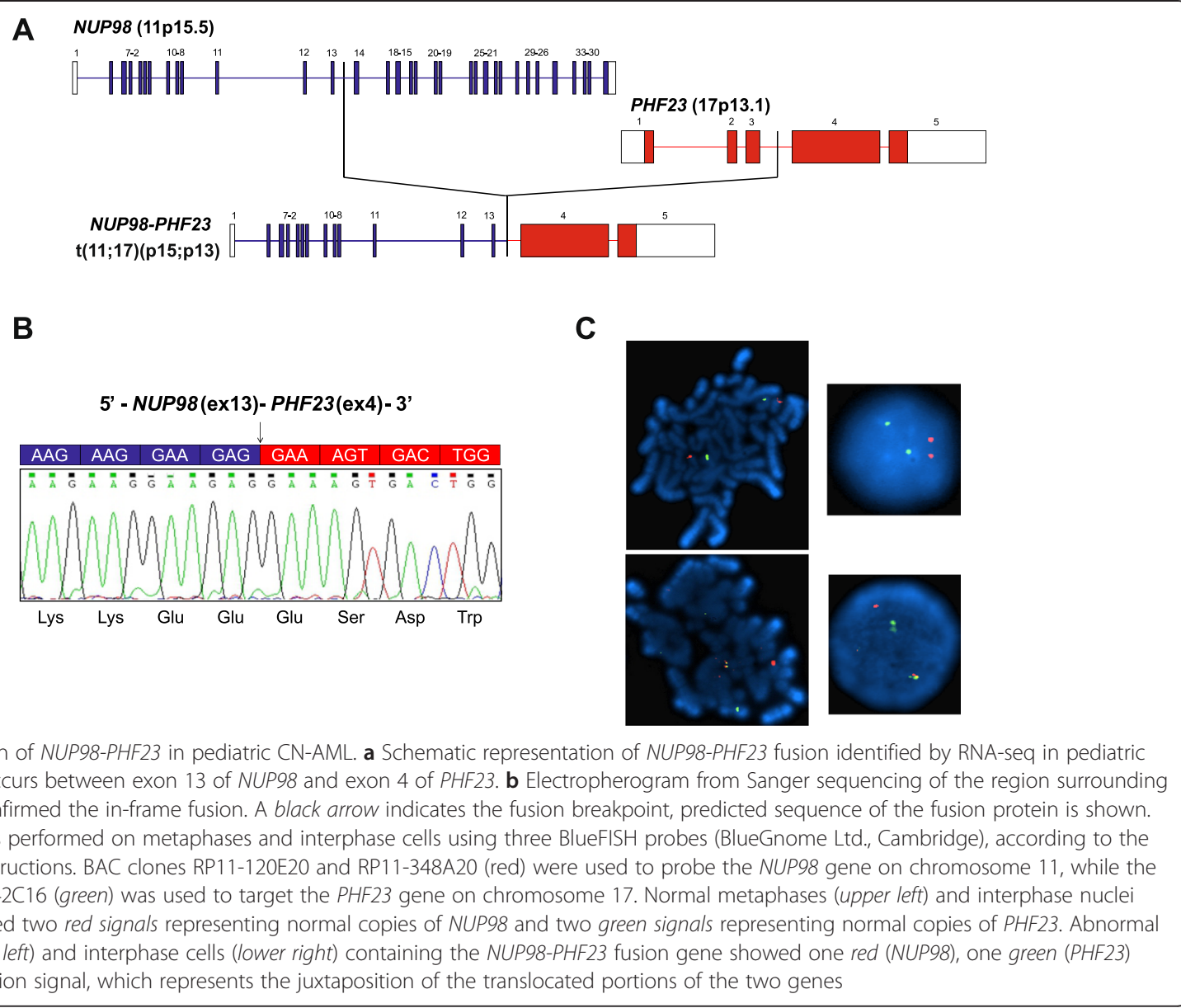

positive by PCR to NUP98-PHF23 fusion gene. Overall, 2 out of $139 \mathrm{CN}$-AML cases were found to harbor NUP98-PHF23 (Table 1). NUP98-PHF23 was not found in any patient harboring FLT3-ITD. Fluorescence in-situ hybridization confirmed the cryptic chromosomal translocation $\mathrm{t}(7 ; 11)(\mathrm{p} 15 ; \mathrm{p} 13)$ leading to the fusion between NUP98 and PHF23 in all cases (Fig. 1c).

So far, many NUP98-rearrangements have been found to be associated with both de novo and therapy-related AML but also with T-cell acute lymphoblastic leukemia with over 28 different partner genes [6]. Recently, the fusion NUP98-JARID1A has been described to be a recurrent event in pediatric acute megakaryoblastic leukemia (11\%), with a distinct HOX gene-expression pattern [7].

Conversely, chromosomal rearrangements and/or mutations of PHF23 have never been previously described in children with AML. Located on the reverse strand of 17p13.1, PHF23 encodes for a protein containing a plant homeodomain (PHD) finger [8] involved in chromatin remodeling [3]. Expression of NUP98-PHF23 has been demonstrated to impair the differentiation of myeloid progenitor cells and promote leukemia development in vitro

Table 1 Clinical features of pediatric CN-AML patients harboring the NUP98-PHF23 fusion gene

\begin{tabular}{|c|c|c|c|c|c|c|c|c|c|c|c|}
\hline Id & Age, years & Gender & $\mathrm{WBC}, \times 10^{9} / \mathrm{L}$ & $F A B$ & $\begin{array}{l}\text { BM blast, } \\
\% \text { at diagnosis }\end{array}$ & $\begin{array}{l}\text { Extramedullary } \\
\text { involvement }\end{array}$ & HSCT (type) & $\begin{array}{l}\text { CR after } \\
\text { induction } \\
\text { therapy }\end{array}$ & $\begin{array}{l}\text { Relapse } \\
\text { (site) }\end{array}$ & $\begin{array}{l}\text { Disease-free } \\
\text { duration } \\
\text { (months) }\end{array}$ & $\begin{array}{l}\text { Survival } \\
\text { duration } \\
\text { (months) }\end{array}$ \\
\hline CN-AML_54 & 2.9 & M & 187 & M1 & 90 & No & Yes (AUTO) & Yes & Yes (BM) & 5 & $30^{\mathrm{b}}$ \\
\hline CN-AML_66 ${ }^{a}$ & 9.0 & M & 1.2 & MO & 70 & No & Yes (MUD) & Yes & - & 65 & 66 \\
\hline CN-AML_3 & 9.7 & M & 6.9 & M4 & 40 & No & Yes (MUD) & Yes & - & 40 & 41 \\
\hline CN-AML_4 & 7.0 & M & 1.8 & M5A & 54 & No & Yes (AUTO) & Yes & - & 103 & 104 \\
\hline
\end{tabular}

AUTO autologous, CR complete remission, HSCT hematopoietic stem cell transplantation, MUD matched unrelated donor, BM bone marrow, WBC white blood cells a patients identified by RNA-seq

${ }^{b}$ dead patient 
and in vivo [8-10]. Cells expressing NUP98-PHF23 are sensitive to disulfiram, an FDA-approved drug, demonstrating the feasibility of targeting this oncoprotein [9].

In summary, we identified, for the first time in childhood AML, a NUP98-PHF23 fusion, demonstrating that this genomic aberrancy is not exceptional (tentative frequency of $2.6 \%$ ) in pediatric CN-AML. These findings enforce the role of epigenetic regulators in pediatric AML and suggest novel therapeutic targets for this disease.

\section{Competing interests}

The authors declare that they have no competing interests.

\section{Authors' contributions}

MT performed the research, coordinated the work, analyzed data, and wrote the paper. RM coordinated the work, analyzed data, and wrote the paper. MT and AA performed the whole-transcriptome massively parallel sequencing. VI performed bioinformatics analyses. MP, EM, and VB performed the screening in the validation cohort. DZ collected and analyzed clinical data. SS performed the cytogenetic analyses. GB, AP, and FL designed and supervised the research. CR and FL equally contributed to the critical revision and writing of the manuscript. All authors read and approved the final version of the manuscript.

\section{Acknowledgements}

This work was supported by grants from Fondazione Ginevra Caltagirone and Fondazione Umberto Veronesi (Milan), by Cariparo IRP-Istituto di Ricerca Pediatrica-Città della Speranza (Padova) and from AIRC (Associazione Italiana Ricerca sul Cancro), special grant 5x1.000 to FL. We acknowledge the contribution of Dr. Anna Leslz for cytogenetic analysis and Maria Grazia Giacometti and Katia Polato for sample preparations.

\section{Author details}

"Department of Pediatrics, "Lalla Seràgnoli" Hematology-Oncology Unit, University of Bologna, Bologna, Italy. ${ }^{2}$ Department of Paediatric Haematology, University of Padova, Padova, Italy. ${ }^{3}$ Giorgio Prodi Cancer Research Centre, University of Bologna, Bologna, Italy. ${ }^{4}$ Department of Pediatrics, San Gerardo Hospital, University of Milano-Bicocca, Monza, Italy. ${ }^{5}$ Department of Pediatric Hematology-Oncology, IRCCS Ospedale Bambino Gesù, Roma - University of Pavia, Pavia, Italy.

Received: 29 March 2015 Accepted: 3 June 2015

Published online: 12 June 2015

\section{References}

1. Tarlock K, Meshinchi S. Pediatric acute myeloid leukemia: biology and therapeutic implications of genomic variants. Pediatr Clin North Am. 2015;62(1):75-93. doi:10.1016/j.pcl.2014.09.007.

2. Masetti R, Pigazzi M, Togni M, Astolfi A, Indio V, Manara E, et al. CBFA2T3-GLIS2 fusion transcript is a novel common feature in pediatric, cytogenetically normal AML, not restricted to FAB M7 subtype. Blood. 2013;121 (17):3469-72. doi:10.1182/blood-2012-11-469825.

3. Reader JC, Meekins JS, Gojo I, Ning Y. A novel NUP98-PHF23 fusion resulting from a cryptic translocation $\mathrm{t}(11 ; 17)(\mathrm{p} 15 ; \mathrm{p} 13)$ in acute myeloid leukemia. Leukemia. 2007;21(4):842-4. doi:10.1038/sj.leu.2404579.

4. Pession A, Masetti R, Rizzari C, Putti MC, Casale F, Fagioli F, et al. Results of the AIEOP AML 2002/01 multicenter prospective trial for the treatment of children with acute myeloid leukemia. Blood. 2013;122(2):170-8. doi:10.1182/blood-2013-03-491621.

5. Pigazzi M, Manara E, Bisio V, Aveic S, Masetti R, Menna G, et al. Screening of novel genetic aberrations in pediatric acute myeloid leukemia: a report from the AIEOP AML-2002 study group. Blood. 2012;120(18):3860-2. doi:10.1182/blood-2012-09-454454.

6. Gough SM, Slape Cl, Aplan PD. NUP98 gene fusions and hematopoietic malignancies: common themes and new biologic insights. Blood. 2011;118(24):6247-57. doi:10.1182/blood-2011-07-328880.

7. de Rooij JD, Hollink IH, Arentsen-Peters ST, van Galen JF, Berna Beverloo H, Baruchel A, et al. NUP98/JARID1A is a novel recurrent abnormality in pediatric acute megakaryoblastic leukemia with a distinct HOX gene expression pattern. Leukemia. 2013;27(12):2280-8. doi:10.1038/leu.2013.87.
8. Reader JC, Leng Q, Rassool FV, Ning Y. Regulation of differentiation by a PHD domain in the NUP98-PHF23 fusion protein. Leuk Res. 2010;34(8):1094-7. doi:10.1016/j.leukres.2010.02.015.

9. Gough SM, Lee F, Yang F, Walker RL, Zhu YJ, Pineda M, et al. NUP98-PHF23 is a chromatin-modifying oncoprotein that causes a wide array of leukemias sensitive to inhibition of PHD histone reader function. Cancer Discov. 2014;4(5):564-77. doi:10.1158/2159-8290.CD-13-0419.

10. Wang GG, Song J, Wang Z, Dormann HL, Casadio F, Li H, et al. Haematopoietic malignancies caused by dysregulation of a chromatin-binding PHD finger. Nature. 2009;459(7248):847-51. doi:10.1038/nature08036.

\section{Submit your next manuscript to BioMed Central and take full advantage of:}

- Convenient online submission

- Thorough peer review

- No space constraints or color figure charges

- Immediate publication on acceptance

- Inclusion in PubMed, CAS, Scopus and Google Scholar

- Research which is freely available for redistribution

Submit your manuscript at www.biomedcentral.com/submit
C Biomed Central 